\title{
Erratum
}

\section{Erratum to "Data Mining of the Thermal Performance of Cool-Pipes in Massive Concrete via In Situ Monitoring"}

\author{
Zheng Zuo, ${ }^{1}$ Yu Hu, ${ }^{1}$ Qingbin Li, ${ }^{1}$ and Liyuan Zhang ${ }^{2}$ \\ ${ }^{1}$ State Key Laboratory of Hydroscience and Engineering, Department of Hydraulic Engineering, Tsinghua University, \\ Beijing 100084, China \\ ${ }^{2}$ Planning and Design Institute of Water Transportation, Beijing 100007, China
}

Correspondence should be addressed to Yu Hu; yu-hu@mail.tsinghua.edu.cn

Received 9 July 2014; Accepted 14 August 2014; Published 28 August 2014

Copyright (C) 2014 Zheng Zuo et al. This is an open access article distributed under the Creative Commons Attribution License, which permits unrestricted use, distribution, and reproduction in any medium, provided the original work is properly cited.

The unit of $Y$-axis in Figure 14 is not $\mathrm{kJ} / \mathrm{min}$ actually, so the number of labels of $Y$-axis is not correct. Here we modify the figure by using $\mathrm{kJ} / \mathrm{min}$ as the unit, as shown in what follows. The curvilinear trends of the curves are not changed. Discussions of the result in the paper are not affected by the correction.

We also take this opportunity to correct two expression errors which may cause confusion to the readers. The line "The heat energy loss from water..." above (1) should be corrected as "The heat energy gained by water..." The line "The heat energy income for the concrete..." above (2) should be corrected as "The heat energy loss from the concrete...".

\section{Acknowledgment}

We are very grateful to Liu Guowei for pointing out the errors. 

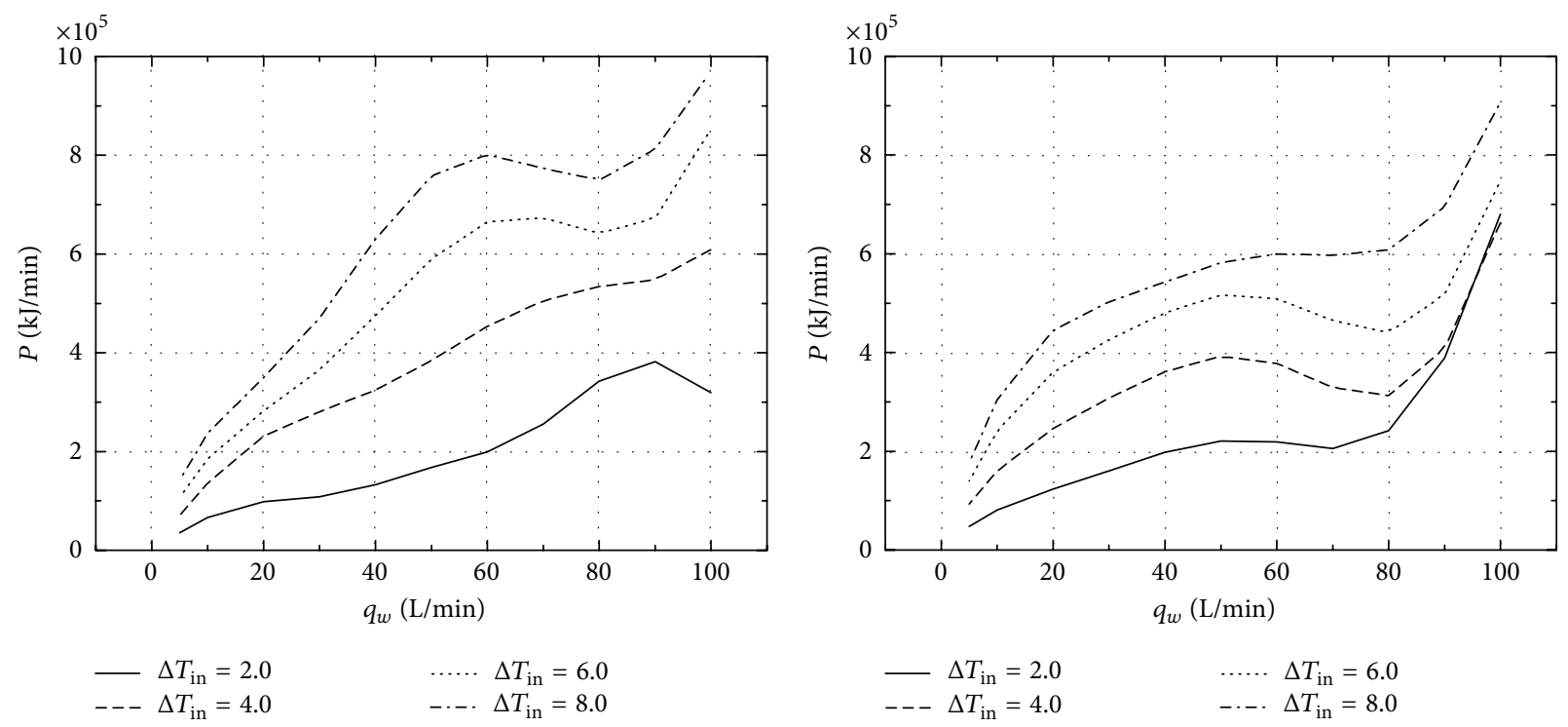

(a) Absorbed power curve of HDPE

(b) Absorbed power curve of iron pipes

FIgURE 14: Absorbed power performance. 


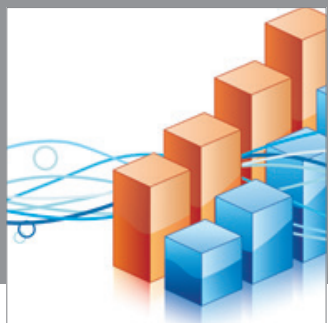

Advances in

Operations Research

mansans

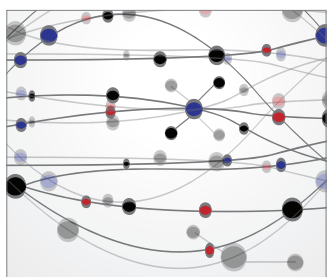

The Scientific World Journal
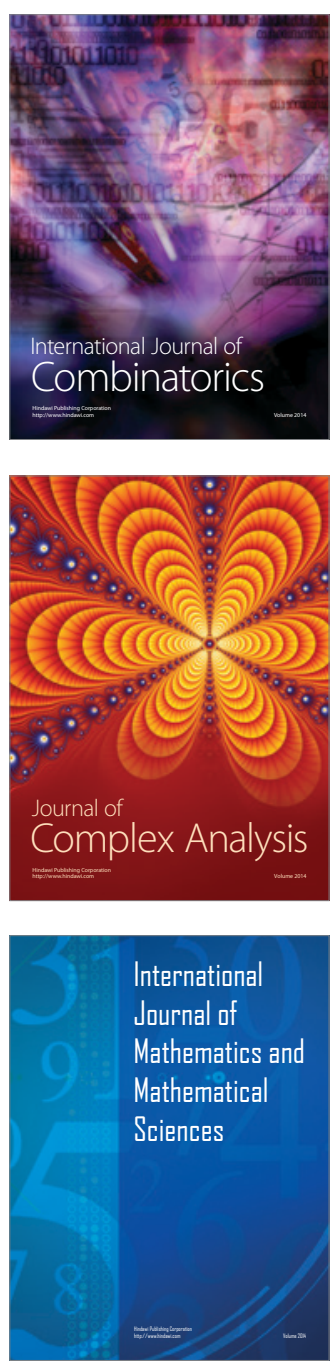
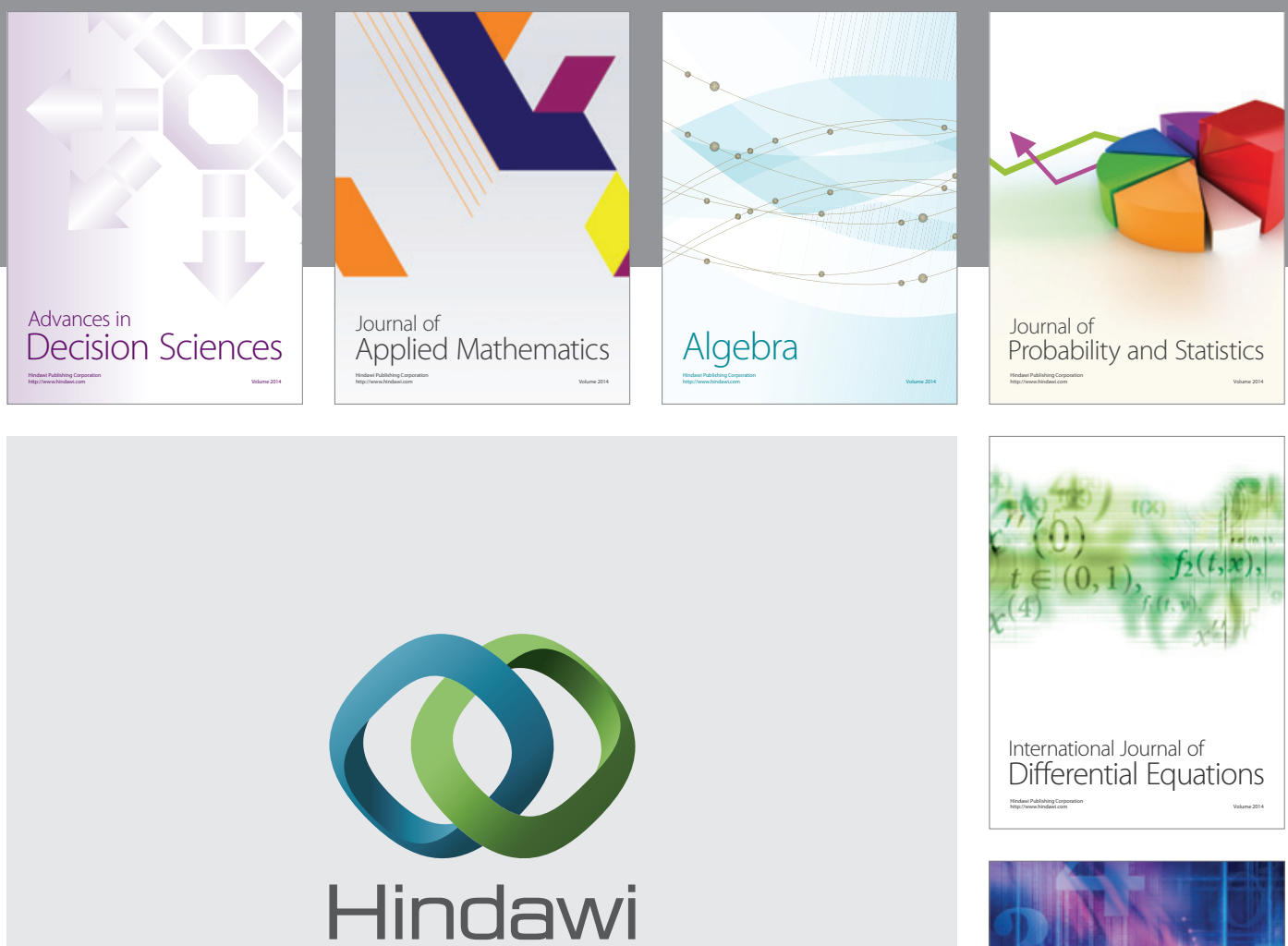

Submit your manuscripts at http://www.hindawi.com
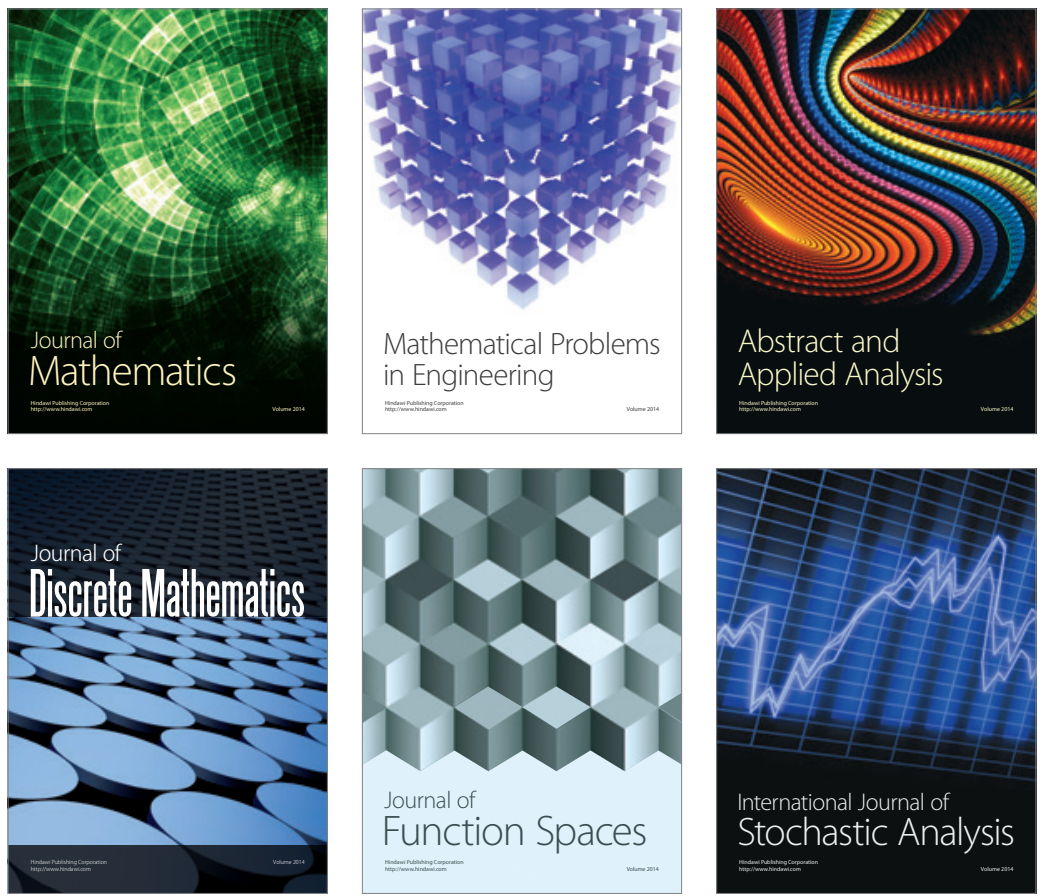

Journal of

Function Spaces

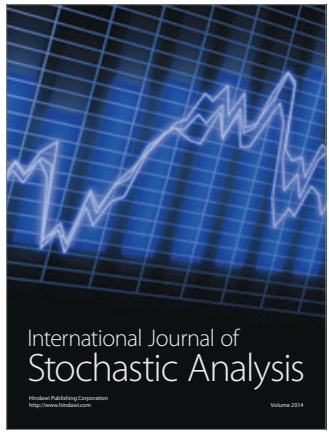

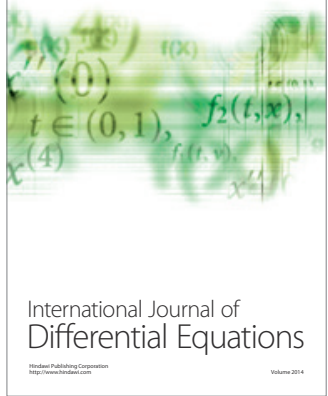
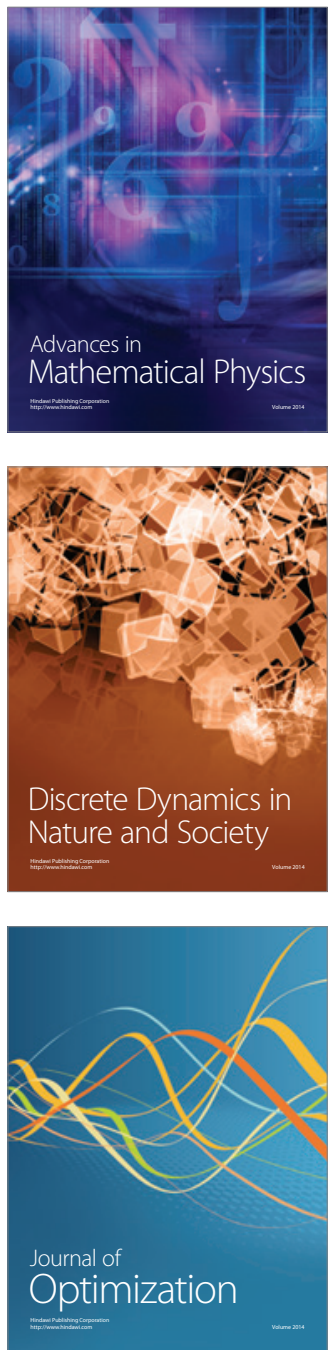